\title{
A Compact, Continuous Adiabatic Demagnetization Refrigerator with High Heat Sink Temperature
}

\author{
P.J. Shirron, E.R. Canavan, M.J. DiPirro, M. Jackson, and J.G. Tuttle \\ Code 552 \\ NASA/Goddard Space Flight Center \\ Greenbelt, MD 20771 \\ USA
}

\begin{abstract}
In the continuous adiabatic demagnetization refrigerator (ADR), the existence of a constant temperature stage attached to the load breaks the link between the requirements of the load (usually a detector array) and the operation of the ADR. This allows the ADR to be cycled much faster, which yields more than an order of magnitude improvement in cooling power density over single-shot ADRs. Recent effort has focused on developing compact, efficient higher temperature stages. An important part of this work has been the development of passive gas-gap heat switches that transition (from conductive to insulating) at temperatures around $1 \mathrm{~K}$ and $4 \mathrm{~K}$ without the use of an actively heated getter. We have found that by carefully adjusting available surface area and the number of ${ }^{3} \mathrm{He}$ monolayers, gas-gap switches can be made to operate passively. Passive operation greatly reduces switching time and eliminates an important parasitic heat load. The current four stage ADR provides $6 \mu \mathrm{W}$ of cooling at $50 \mathrm{mK}(21 \mu \mathrm{W}$ at $100 \mathrm{mK}$ ) and weighs less than $8 \mathrm{~kg}$. It operates from a $4.2 \mathrm{~K}$ heat sink, which can be provided by an unpumped He bath or many commercially available mechanical cryocoolers. Reduction in critical current with temperature in our fourth stage $\mathrm{NbTi}$ magnet presently limits the maximum temperature of our system to $\sim 5 \mathrm{~K}$. We are developing compact, low-current $\mathrm{Nb}_{3} \mathrm{Sn}$ magnets that will raise the maximum heat sink temperature to over $10 \mathrm{~K}$.
\end{abstract}

\section{INTRODUCTION}

Future space telescopes using large arrays of low temperature detectors will require coolers capable of absorbing microwatts of power at temperatures well below $100 \mathrm{mK}$. Adiabatic demagnetization refrigerators (ADRs) are the logical choice in the space environment because of their high efficiency, lack of dependence on gravity, an ease of control. Single stage, single shot ADRs have been used on sub-orbital missions and will be used on the X-Ray Spectrometer (XRS) on the upcoming Astro-E II mission. However, these missions use superfluid helium dewars to provide the $\sim 1.3 \mathrm{~K}$ heat sink required by single stage ADRs. Such dewars are bulky and heavy, they limit mission life, and their complicated testing and ground handling operations impose a costly burden. Future missions will use mechanical coolers based on the compression and expansion of working fluid (helium) to lift heat from $\sim 6 \mathrm{~K}$ to $>200 \mathrm{~K}$, where it can be easily radiated to space. In principle, one could scale up existing single-stage ADR designs to handle the higher heat loads, lower operating temperatures, and higher heat sink temperature of future missions. However, within the restrictions of long view times and high duty cycle compelled by a space mission, such a system would be unacceptably large and heavy. 
The scaling laws controlling ADR mass were broken with the invention, several years ago, of the continuous ADR (CADR)[1]. The CADR has several ADR stages connected in series through heat switches. The first stage, which is connected to the detector package, remains at the operating temperature. When it is demagnetizing, the heat switch between it and the second stage is open, and it acts like a normal ADR. However, before the magnetic field reaches zero, the second stage is commanded to a temperature lower than the operating temperature and the heat switch is closed. In this situation, the first stage must magnetize to maintain operating temperature. This continues until the magnet is fully charged, when the switch opens and the cycle repeats. While the first stage is demagnetizing, the second stage magnetizes to its upper temperature set point, the third stage demagnetizes to set point slightly below this, and the heat switch between them is closed. In this way, heat is cascaded from one stage to the next. Higher heat sink temperatures can be reached by adding additional stages. The temperature span of each stage is determined primarily by the capabilities of the heat switches.

Note that continuous operation, while useful, is not the primary advantage of the CADR. The long hold times of current ADRs are sufficient. However, in single stage ADRs, detector and ADR operation are tightly coupled. By breaking this coupling, the CADR allows the ADR to be cycled much more rapidly. Because the same amount of heat is lifted in each cycle, power density is inversely proportional to cycle time. This is how the CADR can achieve more than 20 times the useful cooling power of a current flight ADR (XRS), while weighing less than half as much. Another advantage of the flexibility allowed by the CADR concept is that at the final sink, heat can be dumped over a larger fraction of the cycle, and therefore at much lower peak rates. Cryocoolers, unlike helium baths, do not respond well to sudden large spikes of heat.

\section{Higher Temperature Stages}

Recent effort has been focused on the extension of the heat sink temperature up to a range reachable by spaceflight cryocoolers. Figure 1 shows a four stage design that operates from $4.2 \mathrm{~K}$. ADRs that operate from higher temperatures generally require higher magnetic fields. Because magnet mass generally scales as field to the fourth power, it is essential to keep the salt pill as small as possible. Cooling power per unit volume of salt is proportional to the density of magnetic ions. At higher temperatures, materials with a higher ordering temperature, which generally have higher magnetic ion density, can be used. Gadolinium Gallium Garnet, $\mathrm{Gd}_{3} \mathrm{Ga}_{5} \mathrm{O}_{12}$, is a commonly used

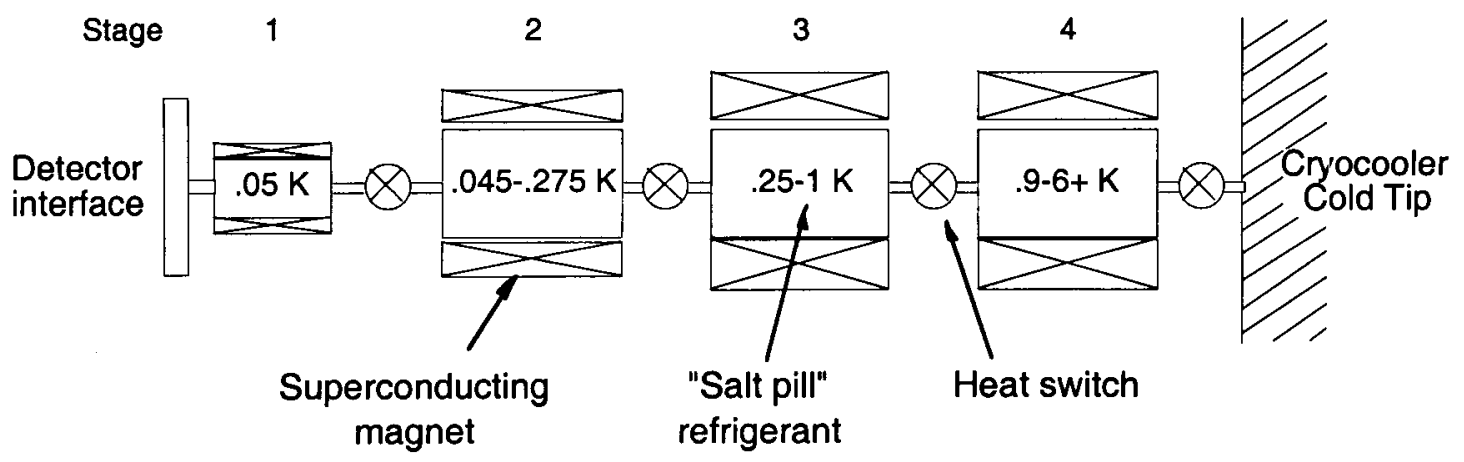

Figure 1. Schematic of four stage CADR. 
compound. It has an ion density $43 \%$ that of pure gadolinium. We have also tested materials with even higher ion density.

Using high ion density materials, the bore of the fourth stage magnet needs to be only $22 \mathrm{~mm}$ ID $\times 35 \mathrm{~mm}$ long. The magnet, which is wound of $75 \mu \mathrm{m}$ multifilamentary $\mathrm{NbTi}$ wire, produces a $4 \mathrm{~T}$ central field with a current of only $3.1 \mathrm{Amp}$, while operating in a vacuum. Low current magnets are necessary for space flight ADRs because low power space flight cryocoolers have limited capacity to absorb the heat conducted through the current leads. Approximately $20 \%$ of the magnetic field is due to enhancement by the silicon-iron shield enclosing the magnet. The shield not only enhances internal fields and reduces stray fields that interfere with other ADR stages; it also makes the internal field more uniform, which reduces temperature gradients in the salt pill. The new magnet can be ramped to full field in $5 \mathrm{~min}$, and ramped back down to zero in $2.5 \mathrm{~min}$.

Older, single shot ADRs, typically used active gas gap heat switches. A sorbent such as charcoal in a small container attached to the main body of the switch through a tube holds the helium. When heated above $\sim 14 \mathrm{~K}$, the sorbent releases the helium, which flows into the narrow gap between the fins attached to either side of the switch and provides a high-conductivity path. When the heater is turned off, the sorbent cools conductively through the tube and readsorbs the helium. In the CADR, unlike single shot devices, the getter heater needs to be on while low temperature stage is running, so radiation from the heater must be shielded. Also, the heat input to the getter eventually gets dumped into the next higher temperature stage, and is a source of inefficiency. Furthermore, the time for the getter to cool down is a limitation on the cycle speed. Finally, each active switch requires leads, a power source, and a controller.

We have eliminated these problems by developing passive gas-gap switches, in which the helium simply adsorbs onto colder surfaces of the switch as the salt pill on that side demagnetizes. We demonstrated this earlier [2] on the switch between stages 2 and 3, where the cold side on that switch reaches temperatures where the vapor pressure even over bulk ${ }^{3} \mathrm{He}$ is negligible. To make this work at higher temperatures, it is necessary to use the higher binding energy of a surface with fractional monolayer ${ }^{3} \mathrm{He}$ coverage. To reduce the ${ }^{3} \mathrm{He}$ coverage while retaining sufficient $\mathrm{He}$ for high on conductance, the surface area of the cold side must be made large. This was done using a disk of sintered stainless steel. By adjusting mass of ${ }^{3} \mathrm{He}$ relative to the surface area, a single switched can be made to have a transition temperature anywhere in the $0.3-6.0 \mathrm{~K}$ range. The conductance is relatively constant above the transition temperature, and falls off rapidly below.

\section{Results}

The four stage system has been tested using an unpumped helium bath $(4.2 \mathrm{~K})$ as the heat sink. Table 1 shows the maximum cooling power as a function of operating temperature. Cooling power is approximately linear with temperature down to $50 \mathrm{mK}$. The thermodynamic efficiency, as a fraction of Carnot efficiency, is $25 \%$, including the effects of magnet hysteresis.। 


\begin{tabular}{cc}
\hline Temperature $(\mathrm{mK})$ & Maximum Cooling Power, $\mu \mathrm{W}$ \\
\hline 50 & 6 \\
60 & 9 \\
70 & 12 \\
80 & 15 \\
90 & 18 \\
100 & 21 \\
\hline
\end{tabular}

Table 1. Maximum cooling power as a function of onerating temnerature.

\section{Future Directions}

In the near future, the four stage system will be moved to a cryostat with a mechanical cryocooler to demonstrate cryogen-free operation. The main concern is ensuring that vibration-induced heat loads on the lower stages are negligible.

We are also working on magnet technology that would allow the CADR to operate from heat sinks up to $10 \mathrm{~K}$. While one or two space prototypes of spaceflight cryocoolers have achieved $4 \mathrm{~K}$, their efficiency is very low (typically $\sim 1 \%$ Carnot). ADRs generally have a higher efficiency. If they could work from a higher heat sink temperature, the efficiency of the entire cryogenic system could be improved. The major impediment has been the availability of high field-to-current magnets. While $\mathrm{Nb}_{3} \mathrm{Sn}$ has a high critical current at $10 \mathrm{~K}$ that would allow the fabrication of a compact $4 \mathrm{~T}$ magnet, traditional fabrication techniques have allowed the use of only large, high current windings. We are working to develop new fabrication techniques that will allow the use of low current $\mathrm{Nb}_{3} \mathrm{Sn}$ magnets that will enable the ADR to operate from a $10 \mathrm{~K}$ heat sink.

\section{References}

1. Shirron, P.J., et al, Advances in Cryogenic Engineering, 45B:1629-1638 (2000).

2. Shirron, P.J., et al, Advances in Cryogenic Engineering, 47:1175-1182 (2002). 


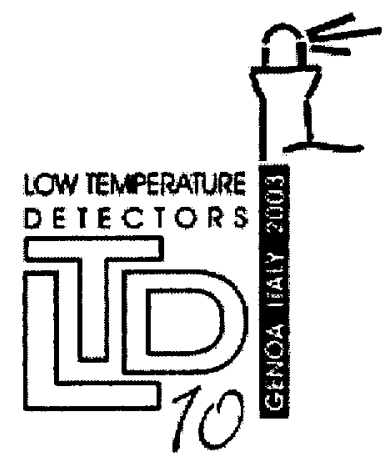

The International Workshops on Low Temperature Detectors are held years and provide an International Forum for assessing the status of knowledge, developments and applications of cryogenic radiation and detectors.

The rapid technical developments of the last years and the outstandil make these detectors very attractive in a variety of fields from basic sciences. Therefore, one of the aims of this workshop is to bring togei detector's scientists and researchers from various fields in order to o future areas of usage.

Dark Matter searches, precise measurements of the Cosmic Microwa Background,

$X$-ray Astronomy and many aspects of the Neutrino Physics are the $n$ topics in which the cryogenic detectors are playing or can play a sign in the near future.

Latest developments open new perspectives in the Energy Dispersive Spectroscopy (EDXRS) as an analysis tool with Microbeams and in $s_{1}$ Spectroscopic Methods at the Synchrotron Radiation. Applications to Microcrystalline Structural Analysis by means of the Beta Decay Fine (BEFS), Biomolecular Mass and Structural Analysis, Biomedics, Arct Environment and Industry are under study.

\section{The topics include:}

- Low Temperature Detector Physics and Technology

- Signal Read-out and Analysis

- Sub-Kelvin Refrigerators for Friendly Use

- Rare Events Detection In Underground Laboratory: Dark MatteI Beta Decay, Rare Nuclear Decays, Solar Neutrino Detection

- Neutrino Physics: Mass, Magnetic Moment, Nuclear Coherent ! neutrino beam

- X-Ray Astronomy: incoming and future Missions for the study $C$ Elemental Abundance, Bulk Motions, Gamma Ray Burst, Interst Intergalactic Medium

- UV to IR Astronomy: single photon spectrophotometry for faint

- Bolometers for Cosmic Microwave Background

- Energy Dispersive X-Ray Spectroscopy at the Synchrotron Radi the Microbeams

- New Methods and new Tools for Analysis in Material Science, I Matter Physics

- Atomic and Plasma Physics 